\title{
Quantitative Investigation of Connections of the Prefrontal Cortex in the Human and Macaque using Probabilistic Diffusion Tractography
}

\author{
Paula L. Croxson, ${ }^{1,3}$ Heidi Johansen-Berg, ${ }^{1}$ Timothy E. J. Behrens, ${ }^{1}$ Matthew D. Robson, ${ }^{2}$ Mark A. Pinsk,, 4 \\ Charles G. Gross, ${ }^{5}$ Wolfgang Richter, ${ }^{4,6}$ Marlene C. Richter, ${ }^{4}$ Sabine Kastner, ${ }^{4,5}$ and Matthew F. S. Rushworth ${ }^{1,3}$ \\ ${ }^{1}$ Centre for Functional Magnetic Resonance Imaging of the Brain, Department of Clinical Neurology and ${ }^{2}$ University of Oxford Centre for Clinical Magnetic \\ Resonance Research, University of Oxford, John Radcliffe Hospital, Headington, Oxford OX3 9DU, United Kingdom, ${ }^{3}$ Department of Experimental \\ Psychology, University of Oxford, Oxford OX1 3UD, United Kingdom, and ${ }^{4}$ Centre for the Study of Mind, Brain, and Behavior and Departments of \\ ${ }^{5}$ Psychology and ${ }^{6}$ Chemistry, Princeton University, Princeton, New Jersey 08544
}

The functions of prefrontal cortex (PFC) areas are constrained by their anatomical connections. There is little quantitative information about human PFC connections, and, instead, our knowledge of primate PFC connections is derived from tracing studies in macaques. The connections of subcortical areas, in which white matter penetration and hence diffusion anisotropy are greatest, can be studied with diffusion-weighted imaging (DWI) tractography. We therefore used DWI tractography in four macaque and 10 human hemispheres to compare the connections of PFC regions with nine subcortical regions, including several fascicles and several subcortical nuclei. A distinct connection pattern was identified for each $\mathrm{PFC}$ and each subcortical region. Because some of the fascicles contained connections with posterior cortical areas, it was also possible to draw inferences about PFC connection patterns with posterior cortical areas. Notably, it was possible to identify similar circuits centered on comparable PFC regions in both species; PFC regions probably engage in similar patterns of regionally specific functional interaction with other brain areas in both species. In the case of one area traditionally assigned to the human PFC, the pars opercularis, the distribution of connections was not reminiscent of any macaque PFC region but, instead, resembled the pattern for macaque ventral premotor area. Some limitations to the DWI approach were apparent; the high diffusion anisotropy in the corpus callosum made it difficult to compare connection probability values in the adjacent cingulate region.

Key words: diffusion-weighted imaging; prefrontal cortex; projections; anatomy; subcortical; macaque MRI

\section{Introduction}

Most knowledge about the anatomical connections of the primate prefrontal cortex (PFC) is derived from tracer studies in the macaque. Speculations about human PFC connections are based on such studies because less is known at first hand about the human PFC. An understanding of the connections of human PFC is important because the pattern of connections of a brain area is a principal determinant of its function (Petrides and Pandya, 2002b). Several distinct anatomical circuits, each with discrete PFC components, have been identified in the macaque, but it is not clear whether the same PFC circuits are present in the human. It has been claimed that there is a disproportionate increase in white matter volume in the human brain (Schoenemann et al., 2005), and so it is possible that the divisions of the

Received April 5, 2005; revised July 4, 2005; accepted Aug. 14, 2005

This work was supported by the Medical Research Council (UK), National Institutes of Health, Whitehall Foundation, Oxford-Princeton Joint Research and Education Committee, Wellcome Trust (P.L.C., H.J.-B.), and Royal Society (M.F.R.). We are grateful to Paul Matthews and Jonathan Cohen for organizing the 0 xford-Princeton collaboration in neuroimaging.

Correspondence should be addressed to Paula Croxson, Department of Experimental Psychology, South Parks Road, 0xford OX1 3UD, UK. E-mail: paula.croxson@psy.ox.ac.uk.

D0I:10.1523/JNEUROSCI.1311-05.2005

Copyright $\odot 2005$ Society for Neuroscience $\quad$ 0270-6474/05/258854-13\$15.00/0 human PFC possess connection patterns distinct from those in the macaque.

Several proposals have been made regarding correspondences between areas of macaque and human PFC on the basis of cytoarchitectural similarities (Chiavaras and Petrides, 2000; Petrides and Pandya, 2002a; Ongur et al., 2003). It is also desirable to know whether human and macaque PFC areas have similar connectivity patterns in accordance with such proposals, which would strengthen the case for treating such areas as similar.

In the present study, we used diffusion-weighted imaging (DWI) and probabilistic tractography to examine patterns of connectivity in the human brain. DWI provides information on the orientation of brain fiber pathways (Basser and Jones, 2002; Beaulieu, 2002). The data are then analyzed with probabilistic tractography techniques that generate estimates of the likelihood of a pathway existing between two brain areas (Behrens et al., 2003b; Hagmann et al., 2003; Parker and Alexander, 2003; Tournier et al., 2003).

The connectivity profiles of $\mathrm{PFC}$ regions were characterized in terms of their probabilities of connection to subcortical areas, including dorsal and ventral striatum, amygdala, and subcortical fascicles connecting PFC to regionally specific posterior areas in temporal and parietal cortex. DWI tractography has previously 


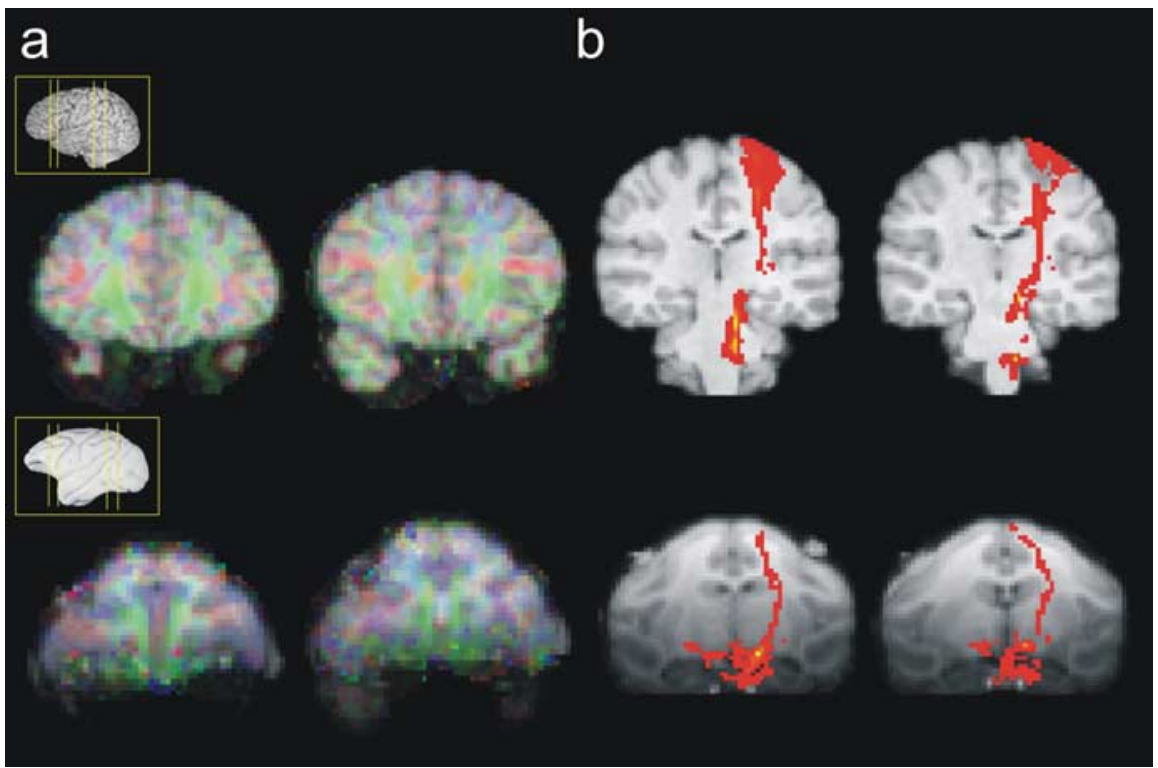

Figure 1. $\quad \boldsymbol{a}$, Trace images overlaid onto structural space illustrating the diffusion fractional anisotropy adjacent to the orbitofrontal cortex. Regions in which diffusion is greatest in the anteroposterior direction are shown in green, those in the dorsoventral axis are shown in blue, and left-right diffusion is shown in red. There is no discernable distortion in either the macaque or the human brain. $\boldsymbol{b}$, Example tract for each brain showing that similar tracts can be traced in both the human and macaque brain. The medial aspect of the corticospinal tract can be seen in both species

proved robust in investigations of subcortical areas in which white matter penetration is greatest (Behrens et al., 2003b; Johansen-Berg et al., 2005).

The main aim of the study was to compare the connections of human PFC identified by DWI tractography with published reports of macaque PFC connections. Human PFC areas possessed distinctive connection patterns with subcortical and cortical regions, and the patterns resembled those seen in the macaque. PFC regions form similar circuits and probably participate in similar regionally specific functional interactions in both species. One area traditionally assigned to human PFC, the pars opercularis (POp), was notable. Its connection probabilities suggested that it resembled macaque premotor rather than macaque prefrontal regions.

In a second experiment, we performed DWI tractography in the macaque. The connectivity patterns for macaque and human were similar, and, despite the lower resolution in the macaque brain scans relative to size, the trends in connectivity were essentially comparable; many of the connections previously found in tracer studies were identified.

\section{Materials and Methods Data acquisition}

We acquired diffusion-weighted data from 10 left hemispheres from healthy human subjects (six male, four female; aged $24-35$ years). Human data were acquired on a 1.5 Tesla Siemens (Erlangen, Germany) Sonata magnetic resonance (MR) scanner with a maximum gradient strength of $40 \mathrm{mT} / \mathrm{m}$. All subjects gave informed written consent in accordance with ethical approval from the Oxford Research Ethics Committee.

We acquired diffusion-weighted data using echo planar imaging (EPI) [ $72 \times 2 \mathrm{~mm}$ thick axial slices; matrix size, $128 \times 104$; field of view (FOV), $256 \times 208 \mathrm{~mm}^{2}$, giving a voxel size of $2 \times 2 \times 2 \mathrm{~mm}$ ]. The diffusion weighting was isotropically distributed along 60 directions with a $b$ value of $1000 \mathrm{~s} / \mathrm{mm}^{2}$. Our 60 diffusion directions were optimized using an electrostatic repulsion model described by Jones et al. (1999). For each set of diffusion-weighted data, we acquired five volumes with no diffusionweighting at points throughout the sequence. We acquired three sets of diffusion-weighted data in total, for subsequent averaging. The total scan time for the DWI protocol was $45 \mathrm{~min}$.

For each subject, we acquired a T1-weighted anatomical image for each subject using a fast, low-angle shot sequence [repetition time (TR), $12 \mathrm{~ms}$; echo time (TE), $5.65 \mathrm{~ms}$; flip angle, 19², with elliptical sampling of $k$-space, giving a voxel size of $1 \times 1 \times 1 \mathrm{~mm}$ in 5:05 $\mathrm{min}$ ].

We acquired diffusion-weighted data from four hemispheres from two adult male macaque monkey brains (Macaca fascicularis). All procedures were approved by the Princeton University Animal Care and Use Committee and conformed to National Institutes of Health guidelines for the humane care and use of laboratory animals. We performed all surgical procedures for the monkeys under strictly aseptic conditions and under general anesthesia with isoflurane (induction, $2-4 \%$; maintenance, $0.5-2 \%$ ) after preanesthetic medication with atropine $(0.08 \mathrm{mg} / \mathrm{kg}, \mathrm{i} . \mathrm{m}$.), ketamine $(2-10$ $\mathrm{mg} / \mathrm{kg}$, i.m.), and acepromazine $(1 \mathrm{mg} / \mathrm{kg})$. The animals had a plastic head bolt for restraining the head, which we implanted to extend vertically from the rostral cranium using ceramic screws and dental acrylic (Pinsk et al., 2005). We treated the animals postsurgically with antibiotics (e.g., Baytril, $2.5 \mathrm{mg} / \mathrm{kg}$, i.m.) and analgesics (e.g., Buprenorphine, $0.01 \mathrm{mg} / \mathrm{kg}$, i.m.), cleaning wound margins of skin surrounding the implant regularly.

During the imaging sessions, we placed the monkeys in the "sphinx" position in an MR-compatible primate chair (Pinsk et al., 2003). We attached a polyetherimide head post to the implanted head bolt of each monkey and secured it to the primate chair to achieve rigid head fixation. We anesthetized the animals with Telazol (tiletamine/zolazepam, $10 \mathrm{mg} /$ kg, i.m.) during scanning.

We acquired images with a 3 Tesla head-dedicated horizontal-bore MR scanner (Magnetom Allegra; Siemens), with a $12 \mathrm{~cm}$ surface coil (model NMSC-023; Nova Medical, Wakefield, MA) for radio frequency transmission and reception. We secured the coil to the head restraint system of the MR-compatible primate chair with two plastic C-clamps above the animal's head.

We acquired a high-resolution $(1.0 \times 0.5 \times 0.5 \mathrm{~mm})$ threedimensional magnetization-prepared rapid-acquisition gradient echo structural scan for each animal (FOV, $128 \times 128$ mm; $256 \times 256$ matrix; $1.0 \mathrm{~mm}$ slice thickness). Diffusion-weighted images were collected using a double spin-echo EPI readout pulse sequence. For each average, we collected images with $1.0 \times 1.0 \times 1.0 \mathrm{~mm}$ resolution using 60 different isotropic diffusion directions ( 45 slices without any gap; FOV, $128 \times 96$ $\mathrm{mm} ; 128 \times 96$ matrix; $1.0 \mathrm{~mm}$ slice thickness; TR, 10,000 ms; TE, $145 \mathrm{~ms}$; interleaved acquisition; two $b$ values of 0 and $1000 \mathrm{~s} / \mathrm{mm}^{2} ; 1056 \mathrm{~Hz} /$ pixel bandwidth). We collected five averages of the data, reversed the phase encode direction, and collected an additional five averages. This allowed us to correct for the susceptibility distortions in the 3 Tesla data (see below, Diffusion-weighted image analysis).

\section{Diffusion-weighted image analysis}

Images acquired from macaque monkey at 3 Tesla field strength suffered more from susceptibility-related distortions than images from human cases that were acquired at 1.5 Tesla field strength. To correct for this difference, we submitted the macaque data to a previously described susceptibility distortion correction method (Munger et al., 2000; Andersson et al., 2004) that relies on the acquisition of two separate datasets with phase encode directions of opposite polarity (see above, Data acquisition). The comparable lack of distortion in the orbitofrontal cortex region is shown for both the human and the macaque in Figure 1, which also shows that similar tracts can be discerned in both species despite the differing acquisition procedures. 
We corrected the human diffusion data for eddy currents and head motion using affine registration to a reference volume (Jenkinson and Smith, 2001). The data from the three acquisitions were subsequently averaged to improve the signal-to-noise ratio.

We performed tissue-type segmentation, skull stripping, and registration on the scans, using tools from the Oxford Centre for Functional Magnetic Resonance Imaging of the Brain's (FMRIB) Software Library (www.fmrib.ox.ac.uk/ fsl). We performed probabilistic tissue-type segmentation and partial volume estimation on the T1-weighted image (Zhang et al., 2001). We thresholded the results to include only voxels estimated at $>35 \%$ gray matter and used this to mask the cortical regions. We skull stripped the diffusion-weighted and T1-weighted images (Smith, 2002) and performed affine coregistration (Jenkinson and Smith, 2001) between the first nondiffusion-weighted volume and the T1-weighted image to derive the transformation matrix between the two spaces. We then calculated probability distributions of fiber direction at each voxel using previously described methods (Behrens et al., 2003a).

We defined nine regions for analysis (seed areas) in each subject's left hemisphere according to anatomical criteria described below. In addition, we defined eight areas within the PFC (target areas). We transformed the masks into the space of each subject's diffusion data using FLIRT (FMRIB's Linear Image Registration Tool) (Jenkinson and Smith, 2001) when necessary.

For each subject, we ran probabilistic tractography from all voxels in each seed mask (Behrens et al., 2003a,b). From each voxel in the mask, samples were drawn from the connectivity distribution, maintaining knowledge of location in structural and DWI spaces, and we recorded the proportion of these samples that passed through each of the cortical target masks as the probability of connection to that zone. We thresholded the results so that only those seed voxels from which $5 \%$ of samples passed through any prefrontal target mask were included in the result.

After thresholding, for each cortical target area, we recorded the number of projections to a prefrontal target as a proportion of the number going to all prefrontal target areas. We then took a mean value for all non-zero voxels in the seed area, giving us the mean proportion of projections to each target area. Taking the proportion of projections allows us to compare the projection patterns of different seed regions, regardless of the overall probability of connections from different areas as assessed on the basis of diffusion anisotropy. Some seed areas, such as some of the fascicles, exhibited higher absolute probabilities of projecting to PFC regions in general than did seed areas in subcortical nuclei such as the amygdala. The higher absolute number of connections in the former case may be a simple consequence of the higher diffusion anisotropy in the white matter compared with the subcortical nuclei.

We visually inspected all generated pathways before intersubject averaging of data. At this stage, we were able to check the anatomical plausibility of the routes of connectivity distributions. In cases in which two cortical regions or two major white matter fascicles were in close contact, we occasionally saw a pathway joining the two areas, although such connections are not anatomically plausible. In these cases, we placed exclusion masks between the areas, for example between the frontal and temporal lobes, and reran the analysis. In one case, we excluded the data from one subject who repeatedly showed such an artifact between two fascicles, in which it was not possible to use an exclusion mask.

To determine whether cortical areas possessed characteristic patterns of connection probability from the subcortical areas considered, we performed repeated-measures ANOVAs on the data, with factors for seed area and target area. Huyhn-Feldt adjustment was applied when necessary.

\section{Definition of seed areas}

We investigated the comparative probability of connections between regions within PFC and a broad range of other brain areas (seed areas) in the human and macaque. By choosing appropriate seed regions, we hypothesized that we would be able to use these patterns of connectivity to dissociate PFC regions from one another in each species and to compare PFC regions between species. To achieve this connectivity-based dissociation of PFC target regions, we chose seed areas that have been shown to have regionally specific connections within macaque PFC and that connect to PFC via distinct white matter fascicles. For example, we investigated PFC connections with temporal lobe cortical areas and PFC connections with the amygdala because the two adjacent temporal lobe regions connect preferentially to somewhat distinct groups of PFC regions (Amaral and Insausti, 1990; Webster et al., 1994; Lavenex et al., 2002; Kondo et al., 2003). While anterior temporal cortical regions project via the uncinate fascicle (UF), amygdala connections travel via a distinct route in the extreme capsule (EC) and are unaffected by uncinate fascicle resection (Amaral and Price, 1984; Ungerleider et al., 1989).

By choosing only subcortical seed areas, we ensured that diffusion tractography could be run robustly from each seed. A series of recent studies have shown that it is possible to use DWI tractography to examine the connections of subcortical white matter tracts and subcortical nuclei (Stieltjes et al., 2001; Behrens et al., 2003b; Lehericy et al., 2004; Johansen-Berg et al., 2005). In general, it may be more difficult to trace connections directly between one cortical seed area and another cortical region, because the relatively low white matter penetration of cortical areas and the high fiber complexity or spreading as fibers reach gray matter can mean that the diffusion anisotropy is too low to initiate reliable probabilistic tractography from the cortex. For this reason, we defined some seed areas in subcortical nuclei, and, to study the PFC connections of remote cortical regions, we did not use seed regions placed directly in the cortex but instead used seed regions in the white matter fascicles associated with particular cortical regions.

The seed areas were chosen to allow us to consider PFC connections with (1) posterior temporal cortex via the EC, (2) anterior temporal cortex via the UF, (3) parietal cortex projections via two divisions of the superior longitudinal fascicle (SLF), SLFII and SLFIII, (4) amygdala, (5) hippocampal connections via the fornix, (6) hippocampal and other medial temporal connections via the cingulum bundle (CB), (7) dorsolateral striatum (STRd), and (8) ventromedial striatum (STRv).

All of the subcortical nuclei masks were defined in a very similar manner in both humans and macaques. In all cases, we selected anatomical points that exist in both species, for consistent definition of the masks. For the fascicle masks, in addition to a priori information from the animal literature, we referred to the fractional anisotropy (FA) map for each individual to identify the likely path and characteristic points of each fascicle. Nine seed masks were identified in the human hemispheres and eight in each of the macaque hemispheres.

\section{Lateral seed areas}

The five lateral seed areas were all relatively close to one another (Fig. 2) but were expected to interconnect with distinct PFC areas.

Uncinate fascicle. Gross dissection of the human brain reveals two major bundles running between the temporal and frontal lobes. Ventrally, the hook-like uncinate fascicle curves anteriorly into the anterior temporal lobe, and, dorsally, it curves into the frontal lobe (Crosby et al., 1962; Smith, 1971). In the macaque, it is known to carry the connections of the more anterior temporal lobe and orbital and ventral PFC (Ungerleider et al., 1989; Petrides and Pandya, 2002a). To examine the connections of the PFC with the more anterior parts of the temporal lobe, we examined the connectivity profile of a seed area in the UF (Figs. 2, 3). The UF was identified, using the FA map, as the point at which the dorsoventrally coursing white matter of the frontal lobe joins to the temporal lobe, as in previous DWI tracking studies (Catani et al., 2002; Mori et al., 2002; Powell et al., 2004). Each mask was $0.6-0.7 \mathrm{~cm}$ long in the anteroposterior plane in the humans and $0.3-0.4 \mathrm{~cm}$ in the macaques.

Extreme capsule. The EC is a distinct fiber bundle dorsal to the UF. It also runs between the temporal and frontal lobes, but its ascent from more posterior temporal lobe regions is shallower than that of the UF. It has also been referred to as the inferior frontoccipital fascicle (Catani et al., 2002; Mori et al., 2002). Because it is not clear that the fiber bundle connects with the occipital lobe, we follow Petrides and Pandya (2002b) and refer to it as the EC pathway. In addition to being distinguishable from the UF, it is also distinct from a separate fiber bundle called the superior occipitofrontal fascicle that is dorsal to the striatum and that may interconnect frontal and parietal regions. In the macaque, the EC interconnects more posterior and superior temporal lobe regions via a pathway through the external capsule (Goldman-Rakic et al., 1984; Pet- 


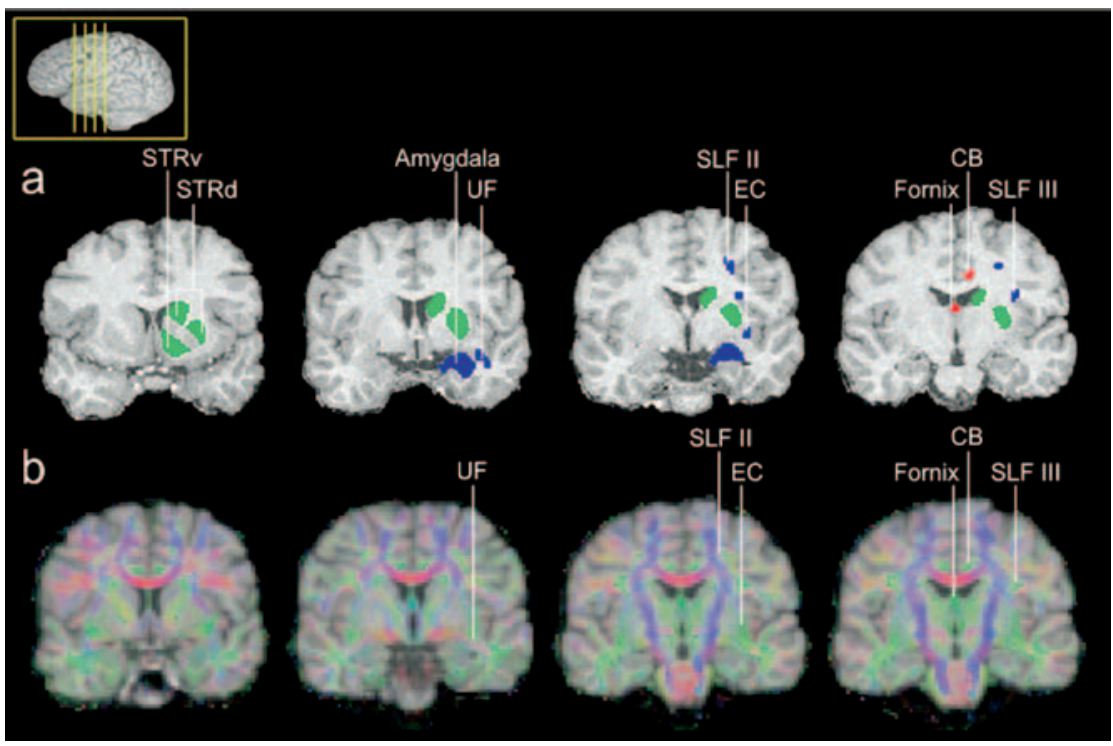

Figure 2. Seed areas were defined according to similar criteria in both species. Examples are shown in the human brain. Lateral seeds are as follows (blue): amygdala; UF, which connects anterior temporal areas to PFC; EC, which connects posterior temporal areas with PFC; and divisions of the superior longitudinal fascicle, SLFIl and SLFIII, which carry fibers from parietal cortex to PFC. Medial seeds are as follows (red): CB and fornix, which connect medial temporal lobe structures, including the hippocampal formation to PFC. Striatal areas are as follows (green): STRd and STRv.

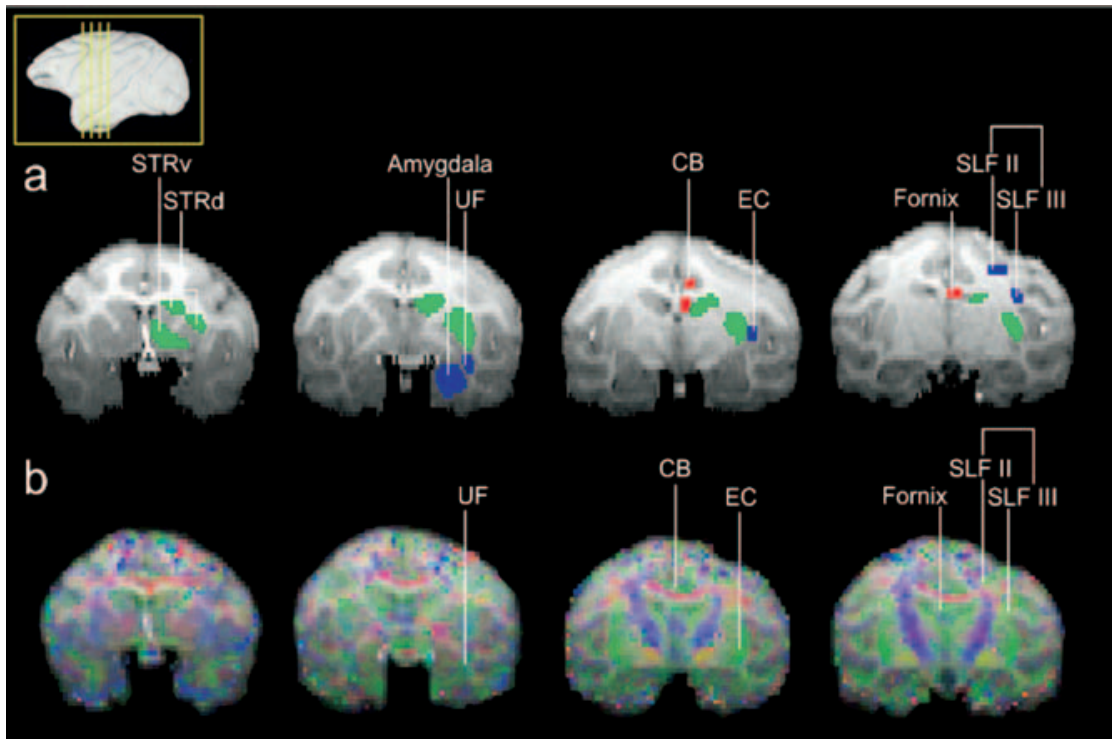

Figure 3. Seed areas were defined according to similar criteria in both species. Examples are shown here in the macaque brain Lateral seeds are as follows (blue): amygdala; UF, which connects anterior temporal areas to PFC; EC, which connects posterior and superior temporal areas with PFC; and divisions of the superior longitudinal fascicle, SLFII and SLFIII, which carry fibers from parietal cortex to PFC (combined into a single mask). Medial seeds are as follows (red): CB and fornix, which connect medial temporal lobe structures, including the hippocampal formation to PFC. Striatal areas are as follows (green): STRd and STRv.

rides and Pandya, 1988, 2002b). We defined the EC by using the FA map to find the anteroposterior fiber bundle, which runs between the superior temporal lobe and the frontal lobe, lateral to the claustrum (Figs. 2, 3). The EC was defined at a point between the gray matter and the claustrum, in which it was distinct from the UF (Petrides and Pandya, 1988; Catani et al., 2002; Mori et al., 2002; Powell et al., 2004). Each mask was 0.6-0.9 $\mathrm{cm}$ long in the anteroposterior plane in the humans and $0.3-0.4 \mathrm{~cm}$ in the macaques.

Superior longitudinal fascicle. The inferior parietal cortex also has extensive connections with the PFC. The connections are conveyed via more than one branch of the SLF, which is a very large fiber bundle with at least three divisions (Petrides and Pandya, 1984, 1988; Makris et al.,
2005). In the human brains, we were able to reliably and consistently identify two distinct divisions that are likely to correspond to SLFI and SLFIII (Petrides and Pandya, 2002b; Makris et al., 2005). In both cases, we used the FA map to conservatively identify anteroposteriorly projecting fibers dorsolateral to the putamen and posterior to the point at which EC fibers from the temporal lobe arrived in the extreme capsule (Fig. 2). The SLFII mask was more dorsal and medial than the SLFIII. In the macaque, the separation between tracts from the two SLF regions was less clear and reliable because of the small size of the brain. Therefore, for the macaques, we defined a single SLF mask comprising both the SLFII and SLFIII components (Fig. 3).

Amygdala. The amygdala is known to have extensive connections with the PFC especially with the orbitofrontal cortex (Porrino et al. 1981; Amaral and Price, 1984). The amygdala interconnections with the PFC remain even after the transection of the more lateral pathways such as the UF that conveys connections from the temporal lobe cortex (Ungerleider et al., 1989), and so it is possible to distinguish the connections of the amygdala with the PFC from other temporal lobe connections. The amygdala was identified in both coronal and sagitta section, ensuring that separation was kept from the hippocampus to avoid confounding results (Weniger et al., 2004) (Figs. 2, 3).

\section{Medial seed areas}

There are several routes by which medial cortical areas, and notably the hippocampus, are connected with the PFC. We examined two distinct routes that could be reliably discerned because of the large and discrete nature of the tracts. The first route is via the fornix (Cavada et al., 2000), and the second route is the $\mathrm{CB}$ (Morris et al., 1999).

Fornix. The fornix provides a connection between the hippocampal formation and orbital areas (Cavada et al., 2000). It lies close to the midline and is distinctive in sagittal and coronal sections running between the lateral and third ventricles (Fig. 2). We defined our mask in the descending part of the fornix in which it is distinct from other midline structures such as the anterior commissure (Figs. 2, 3).

Cingulum bundle. The hippocampus and other regions on the medial surface also connect to the PFC via the CB (Goldman-Rakic et al., 1984; Morris et al., 1999). The CB courses dorsally from the vicinity of the hippocampus and curves round the most caudal point of the corpus callosum, then runs anteriorly, above the corpus callosum toward medial frontal cortex. We identified it using the FA map at a point above the corpus callosum at which the curvature of the fascicle was not too severe (Figs. 2, 3).

\section{Striatal seed areas}

The striatum was separated into the two major areas (Voorn et al., 2004): STRd and STRv. In the macaque, these regions are known to have distinct functions and are known to have different connection patterns with the PFC (Selemon and Goldman-Rakic, 1985, 1988; Kunishio and Haber, 1994; Eblen and Graybiel, 1995; Haber et al., 1995; Ferry et al., 2000). We defined the STRv as the area ventromedial to the internal capsule, with its outer boundary limited by the visible edge of the internal capsule (Fig. 2). 
It included much of the region thought to be the nucleus accumbens in both macaque and human species (Meredith et al., 1996). The remaining area was split in half, and the most dorsolateral part was defined as the STRd (Fig. 2 ). Following the procedure used by Lehericy et al. (2004) in a diffusion imaging study of the human striatum, the central region between the STRd and STRv, which is difficult to unambiguously attribute to either STRd or STRv, was not included in the analysis. The same striatal areas were defined in the macaques (Fig. 3).

\section{Human cortical masks}

We defined PFC cortical masks using reliable anatomical features visible on magnetic resonance imaging (MRI) scans to define regional boundaries. A number of proposals have been made regarding the possible correspondences between areas of the human and macaque frontal lobes (Rajkowska and Goldman-Rakic, 1995; Pandya and Yeterian, 1996; Paus, 1996, 2001; Paus et al., 1996, 2001; Chiavaras and Petrides, 2000; Petrides and Pandya, 2002a; Ongur et al., 2003). Our anatomical boundaries were

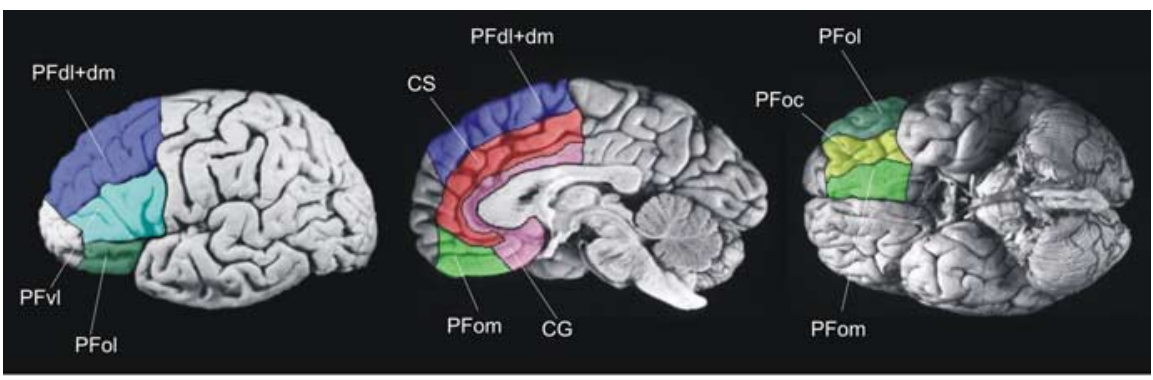
drawn in such a way as to facilitate the comparison of possibly similar areas in the two species.

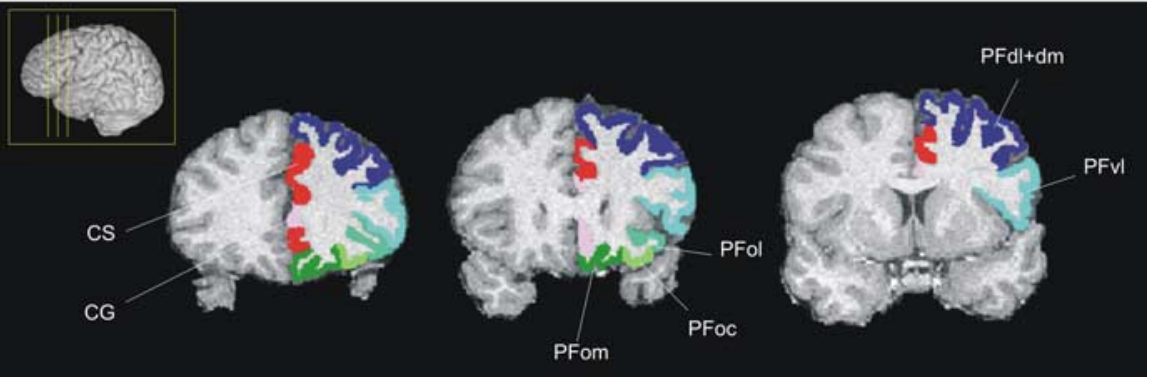

Figure 4. Eight areas were defined within the PFC of 10 human left hemispheres: PFd, PFdl + dm, PFvl, PFol, PFoc, PFom, CS, and CG.

We defined eight PFC zones on the basis of reliable anatomical landmarks. Initially, we defined the PFC region as the area anterior to the superior and inferior precentral sulci on the lateral surface. A vertical plane $6 \mathrm{~mm}$ posterior to the one passing through the anterior commissure $(y=-6$ plane) on each brain when transformed into standard space (Talairach and Tournoux, 1988; Collins et al., 1994) defined the posterior limit of the region investigated on the medial surface. The posterior limit of the investigation on the orbital surface was a line drawn between the posterior extent of the lateral and medial orbital sulci, which was then extended to meet the posterior boundaries on the lateral and medial surfaces. The rostral extent was the frontal poles, which were defined as the point at which the three lateral sulci could no longer be clearly distinguished. The frontal eye fields were also not considered in the analysis. A region that included much of the frontal eye field (Paus, 1996), extending $10 \mathrm{~mm}$ immediately anterior to the meeting of the inferior branch of the superior precentral sulcus and the superior frontal sulcus, was therefore excluded from this area. We defined three large regions that were subsequently divided into smaller PFC subregions (Fig. 4 ). The areas were defined according to the following criteria.

\section{Medial PFC}

This region included the cingulate gyrus (CG) and both banks of the cingulate sulcus (CS) (Vogt et al., 2003). In those subjects possessing a second cingulate sulcus or paracingulate sulcus (common in the left hemisphere), the lower bank of this sulcus was also included (Vogt et al., 1995; Paus, 1996; Paus et al., 1996). Subcallosally, the region extended ventrally to include the cingulate sulcus and, following Ongur et al. (2003), the subcallosal region in which areas $32 \mathrm{pl}$ and 25 are found. The region is indicated by the red areas in Figure 4.

The medial prefrontal cortex region was further split into CG and CS regions (Fig. 4). The CG was defined first and included tissue ventral and posterior to the lower bank of the CS, including the rostral cingulate motor zone and the tissue immediately anterior with which it is anatomically interconnected and functionally inter-related (Van Hoesen et al., 1993; Paus, 2001; Picard and Strick, 2001; Procyk and Joseph, 2001; Matsumoto et al., 2003; Walton et al., 2004). The remaining tissue in the medial frontal region was designated as CS. In some subjects, the CS region therefore just included both banks of the cingulate sulcus, but, in subjects with a paracingulate sulcus, it extended to include the superior cingulate gyrus and the lower bank of the paracingulate sulcus.

\section{Lateral and dorsomedial PFC}

This region extended from the upper bank of the paracingulate sulcus (or from dorsal to the cingulate sulcus in those subjects not possessing a paracingulate sulcus) to the horizontal ramus of the lateral fissure (Fig. 4, blue).

Two areas were defined within the lateral PFC region: ventrolateral PFC (PFvl) and a combined dorsolateral and dorsomedial area $(\mathrm{PFdl}+\mathrm{dm})$. PFvl extended from the horizontal ramus of the lateral fissure to the inferior frontal sulcus. PFdl $+\mathrm{dm}$ included the middle frontal gyrus and superior frontal gyrus, extending from the inferior frontal gyrus to the cingulate or paracingulate sulcus. The frontal eye fields were not included in this study because it is not clear whether this region should be considered prefrontal. Von Bonin and Bailey (1947) argued for it being a transitional area between prefrontal and premotor cortex. More recently, other anatomists, such as Pandya and Yeterian (1996), used the term premotor to refer to both area 8 and area 6 . Functionally, there are a number of similarities between areas 6 and 8; lesions in these areas have quite distinct effects to more anterior dorsolateral prefrontal lesions, and, in both cases, the lesions particularly disrupt aspects of response selection (Passingham, 1995; Petrides, 2005). A full detailed comparison of the various subregions of the premotor and motor cortices is beyond the remit of the current study.

An additional analysis was also performed to compare the most posterior PFvl area with its more anterior parts of PFvl because the initial results suggested that posterior $\mathrm{PFvl}$ had a distinct pattern of connectivity with several fascicles. The posterior PFvl area was referred to as the pars opercularis. We used a conservative estimate of the extent of the POp that was based on the description of area 44 in the POp by Amunts et al. (1999), who reported that the border between areas 44 and 45 was located either near the vertical branch of the lateral fissure or the diagonal sulcus, when present, although there was much intersubject variation in exact location of the boundary. Moreover, there is variability between individuals in the prominence of the diagonal sulcus. We therefore took the first sulcus anterior to the inferior precentral sulcus to be the anterior border of the POp and the inferior precentral sulcus to be the posterior border of POp. All of the tissue in the POp mask was therefore likely to consist of tissue that would be described as belonging to area 44 .

\section{Orbitofrontal cortex}

This region extended from the horizontal ramus of the lateral fissure, across the orbital surface and onto the medial surface to include the gyrus ventral to the cingulate sulcus and the subcallosal cingulate areas (Chiavaras and Petrides, 2000; Ongur et al., 2003). On the medial surface, 


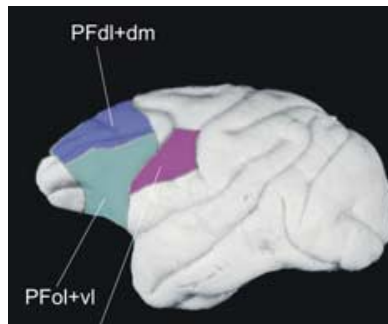

PMv

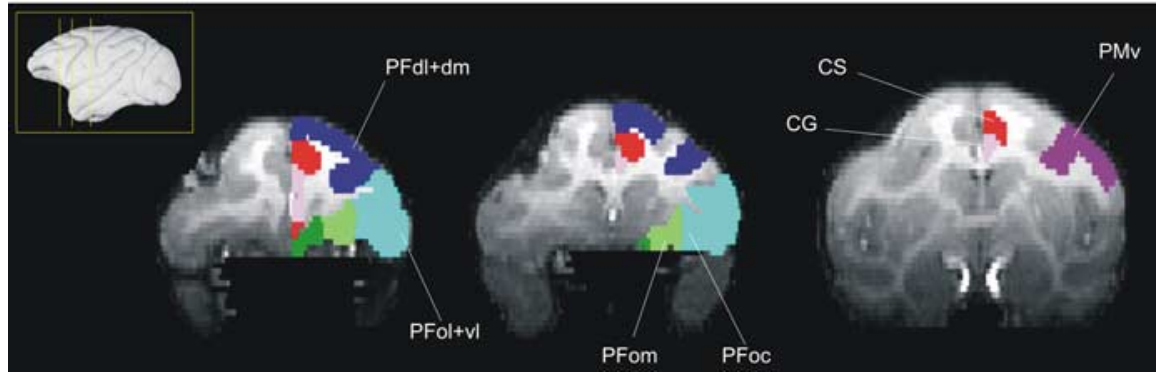

Figure 5. Eight areas were defined within the macaque PFC: PFd, PFdl $+d m$, PFol + vl, PFoc, PFom, CS, CG. We also examined the connections of the PMv. prising the gyrus rectus and medial orbital gyrus. PFoc was the gray matter between the medial orbital sulcus to the lateral orbital sulcus.

\section{Lateral and dorsomedial PFC}

This region included the remaining tissue extending from the cingulate sulcus to the lateral orbital sulcus. This was then further subdivided into two areas. First, a combined ventrolateral $\mathrm{PFC}$ and lateral orbital area $(\mathrm{PFol}+\mathrm{vl})$ area included the inferior convexity of the ventrolateral prefrontal cortex and extended around to include the tissue lateral to the lateral orbital sulcus. The dorsal boundary of the PFol $+\mathrm{v}$ was on the convexity directly ventral to the sulcus principalis. The second area included all of the tissue of the dorsal convexity and was designated dorsolateral and dorsomedial PFC $(\mathrm{PFdl}+\mathrm{dm})$, beginning just below the principal suclus and extending to just dorsal of the cingulate sulcus on the medial surface.

We defined one additional area in the macaque because our preliminary analyses showed that its connections were similar to those of the posterior part of the human ventrolateral area POp area. We defined a ventral premotor cortex (PMv) region, which included the area anterior to the central sulcus, posterior anterior to the cingulate sulcus, the boundary was from the rostral sulcus to the horizontal ramus of the lateral fissure. The region is indicated by the green areas in Figure 4.

Three areas were defined within the orbitofrontal region: medial orbital (PFom), central orbital (PFoc) and lateral orbital (PFol). PFom included the area from the cingulate sulcus/rostral sulcus to the medial orbital sulcus (thus comprising the gyrus rectus and medial orbital gyrus). PFoc was the central orbital gyrus between the medial orbital sulcus and the lateral orbital sulcus. PFol extended from the lateral orbital sulcus to the horizontal ramus of the lateral fissure and included the lateral orbital gyrus and the pars orbitalis of the inferior frontal gyrus (Chiavaras and Petrides, 2000).

\section{Macaque cortical masks}

As had been the case with the human brains, we selected sulci that were easily and reliably identified on MRI scans to be the boundaries of the macaque PFC areas. In addition, we chose boundaries that would facilitate comparison with the human data; however, a simple one to one correspondence between human and macaque PFC areas was not always possible, particularly in the case of the ventral prefrontal region. In the macaque, this area of PFC included both ventrolateral and lateral orbital tissue. In total, eight PFC areas were distinguished in the macaque brains.

The PFC was defined as the area rostral to the arcuate sulcus. As in the human brains, the frontal eye fields and the frontal poles were not included in the analysis. The frontal poles served as the rostral boundary to the masks, defined as the tissue anterior to the position at which the two orbital sulci could no longer be clearly defined. We defined the frontal eye fields as the area within the bounds of, and forward of, the arcuate sulcus, (not including the principal sulcus itself). Three large PFC masks were first defined and then subdivided into smaller areas (Fig. 5).

\section{Medial PFC}

This region extended from the rostral sulcus to the dorsal bank of the cingulate sulcus on the medial surface. This was then divided into a CS region that included both banks of the cingulate sulcus and a CG region that included the more ventral tissue between the cingulate sulcus and the corpus callosum.

\section{Orbitofrontal cortex}

This region included the tissue between the rostral sulcus and the lateral orbital sulcus. This was then divided into two areas: PFom and PFoc. PFom extended from the rostral sulcus to the medial orbital sulcus, com- to the arcuate sulcus, and below the spur of the arcuate sulcus (Rizzolatti et al., 2002).

\section{Results}

\section{Human PFC connections with lateral seed areas}

Example tracts and connection distributions for each lateral seed area (UF, EC, SLFII, SLFIII, and amygdala) are summarized in Figure 6. Comparison of all five lateral areas by repeatedmeasures ANOVA revealed a significant interaction of the five seed area and eight target areas $\left(F_{(8.142,48.850)}=28.269 ; p<\right.$ $0.001)$, suggesting that the different seed areas had distinct patterns of connection probability with the various $\mathrm{PFC}$ regions. The connection probabilities of each seed region were therefore examined separately.

The highest probability connections of the human UF were with the orbitofrontal cortex, and, within this region, the most prominent connection was with the PFoc (Fig. $6 a$ ). Analysis by repeated-measures ANOVA revealed that the difference between the probability of connection between UF and each of the PFC target regions was significant $\left(F_{(2.844,22.749)}=31.963 ; p<0.001\right)$.

The human EC also had connections with orbitofrontal cortex areas, particularly PFoc and PFol. Within the orbitofrontal cortex, the connections had a more lateral bias, toward area PFol, than was the case for the UF. The most probable connections, however, were with the more ventrolateral area PFvl (Fig. 6b). The differences in the probabilities of the EC connections with the various $\mathrm{PFC}$ areas were significant $\left(F_{(2.651,21.209)}=7.762 ; p<\right.$ 0.005).

The human SLFII (Fig. 6c) had the highest probability of connection with $\mathrm{PFdl}+\mathrm{dm}$ and also was connected with high probability, albeit to a lesser extent, with PFd. Again, there were significant differences in the probabilities of seed area connections with each PFC area $\left(F_{(1.008,6.050)}=266.343 ; p<0.001\right)$. The human SLFIII (Fig. $6 d$ ) showed a very high probability of connection with the PFvl $\left(F_{(1.176,9.412)}=272.426 ; p<0.001\right)$.

The final lateral area, the amygdala, had its highest probability PFC connectivity with the orbitofrontal cortex. The connection pattern within the orbitofrontal cortex, however, was quite distinct 
from that seen for either the UF or the EC because it was biased to the more medial area, PFom, in addition to PFoc (Fig. 6e). As with the other seed areas, there were significant differences in the probability of seed areas connections with each PFC area $\left(F_{(4.781 \text {, }}\right.$ 43.033) $=7.382 ; p<0.001$.

\section{Human lateral connections within the PFvl}

For both the SLFIII and EC, the highest probabilities of connections were with the PFvl. To more clearly define the connections of the posterior and superior temporal lobe and the parietal lobe with the PFC, it was of interest to determine whether these two regions terminated in the same part of the PFvl. To do this, we examined the peak projection point of both the SLFIII and the EC fascicles in the PFvl mask of each human subject. To normalize the results, we transformed the resulting coordinates into Talairach space using a registration matrix for each subject obtained by performing registration between each subject's T1-weighted scan and a standardized template (Passingham et al., 2002). The resulting coordinates were plotted (Fig. 7a) and analyzed statistically. A repeated-measures ANOVA with fascicle (two levels) and coordinate of peak tract position [three levels: mediolateral $(X)$, anteroposterior $(Y)$, and dorsoventral $(Z)$ ] revealed that the distributions were separate in the dorsoventral, anteroposterior, and mediolateral axes. There was a significant interaction between fascicle and coordinate position $\left(F_{(2,16)}=\right.$ 28.14; $p<0.001)$, and paired-samples $t$ tests confirmed the differences along each axis $(p<0.05)$. As can be seen in Figure $7 a$, the SLFIII terminates in a more posterior, dorsal, and lateral part of the PFvl than does the EC.

To further analyze the connections of PFvl in the human brain, we also compared the connections of the posterior part of the PFvl region, POp, and the rest of the PFvl anterior to this region. There was a two-way interaction between the tract origin and PFC area, indicating that POp had a distinct pattern of connectivity from anterior PFvl (Fig. 7b). A relatively high proportion of pathways seeded in SLFIII terminated in POp compared with those from EC and SLFII. In contrast, the highest proportion of pathways terminating in anterior PFvl were seeded in EC as opposed to the other two areas. There was a low probability of SLFII connections to either POp or anterior PFvl; the SLFII connections tended to terminate in more dorsal PFdl + dm areas (Fig. 6).

\section{a UF}
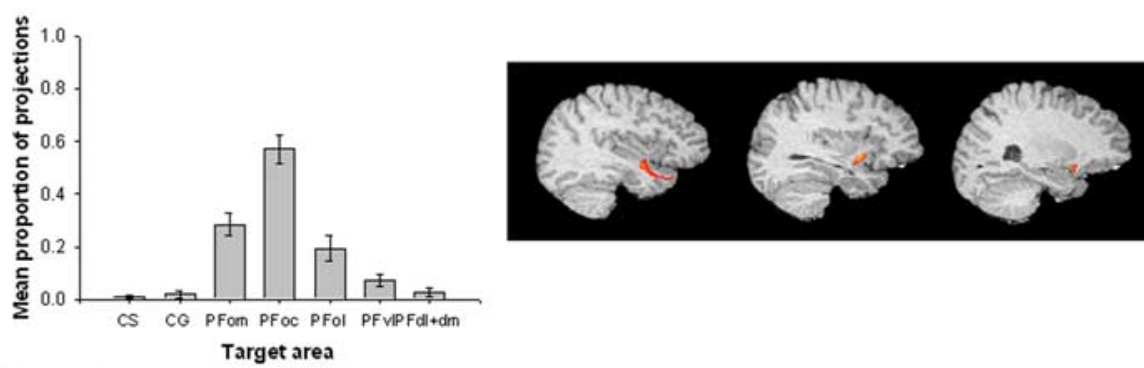

\section{b EC}
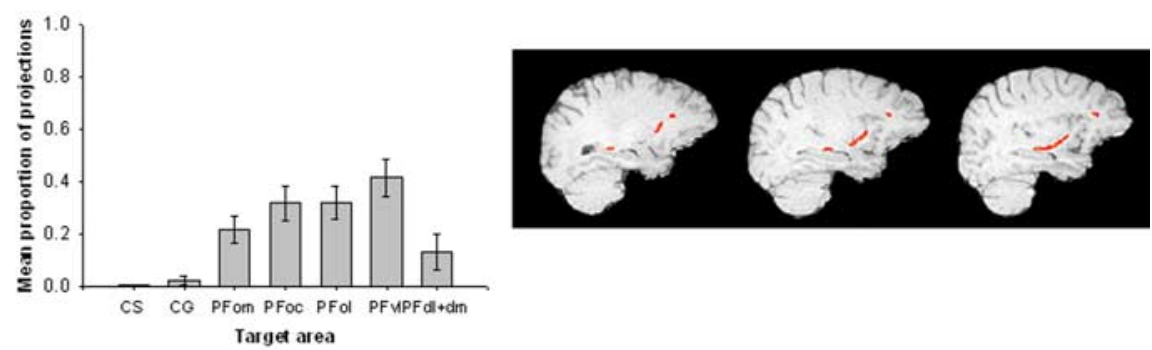

\section{c SLF ॥}
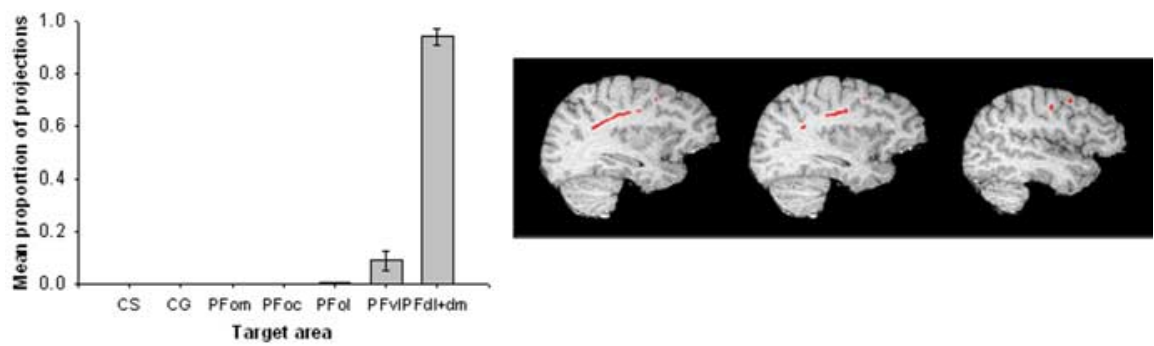

\section{d SLF III}
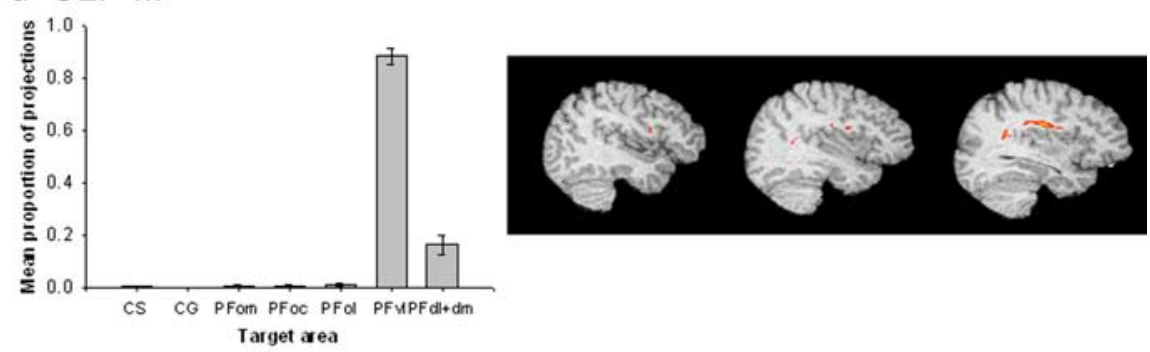

\section{e Amygdala}

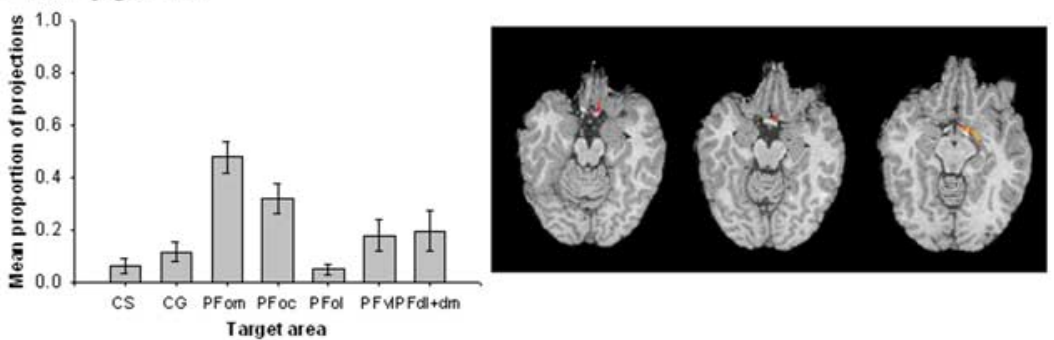

Figure 6. Quantitative results of probabilistic tractography from lateral seed masks in the human brain, and examples of a corresponding tract for each, run from a single seed point. $\boldsymbol{a}$, The majority of connections from anterior temporal lobe via the UF are to orbital PFC, with a greater bias to central orbital areas. $\boldsymbol{b}$, Connections from posterior and superior temporal lobe areas via the EC are mainly to the central/lateral orbital and ventrolateral PFC areas, whereas connections via the SLF, connecting with parietal areas, are most likely to be with the PFvl (for SLFII) and PFdI + dm (in the case of SLFIII), with very low probabilities to orbital areas $(\boldsymbol{c}, \boldsymbol{d}) . e$, The amygdala connections are most likely to be with PFom. 


$$
\text { a }
$$

PFvl connections (axial plane)

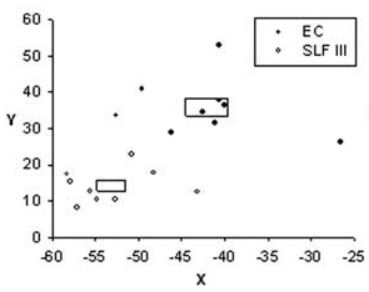

PFvl connections (saggital plane) POp and anterior PFvl connections
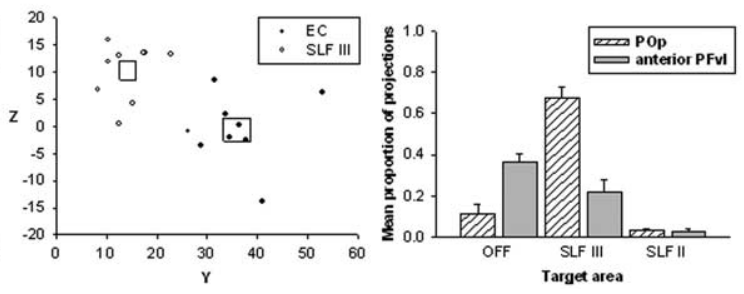

Figure 7. PFvl connections in the human in more detail. $\boldsymbol{a}$, Termination point of tracts seeded in EC and SLFIII in the PFvl in Talairach space in an axial slice and a sagittal slice. Boxes represent SEM for each dataset. Connections from the SLF target a significantly more posterior, dorsal, and lateral part of PFvl than connections from the EC. $\boldsymbol{b}$, Proportion of projections from EC, SLFIII, and SLFIl to human POp and the more anterior remainder of PFvl.
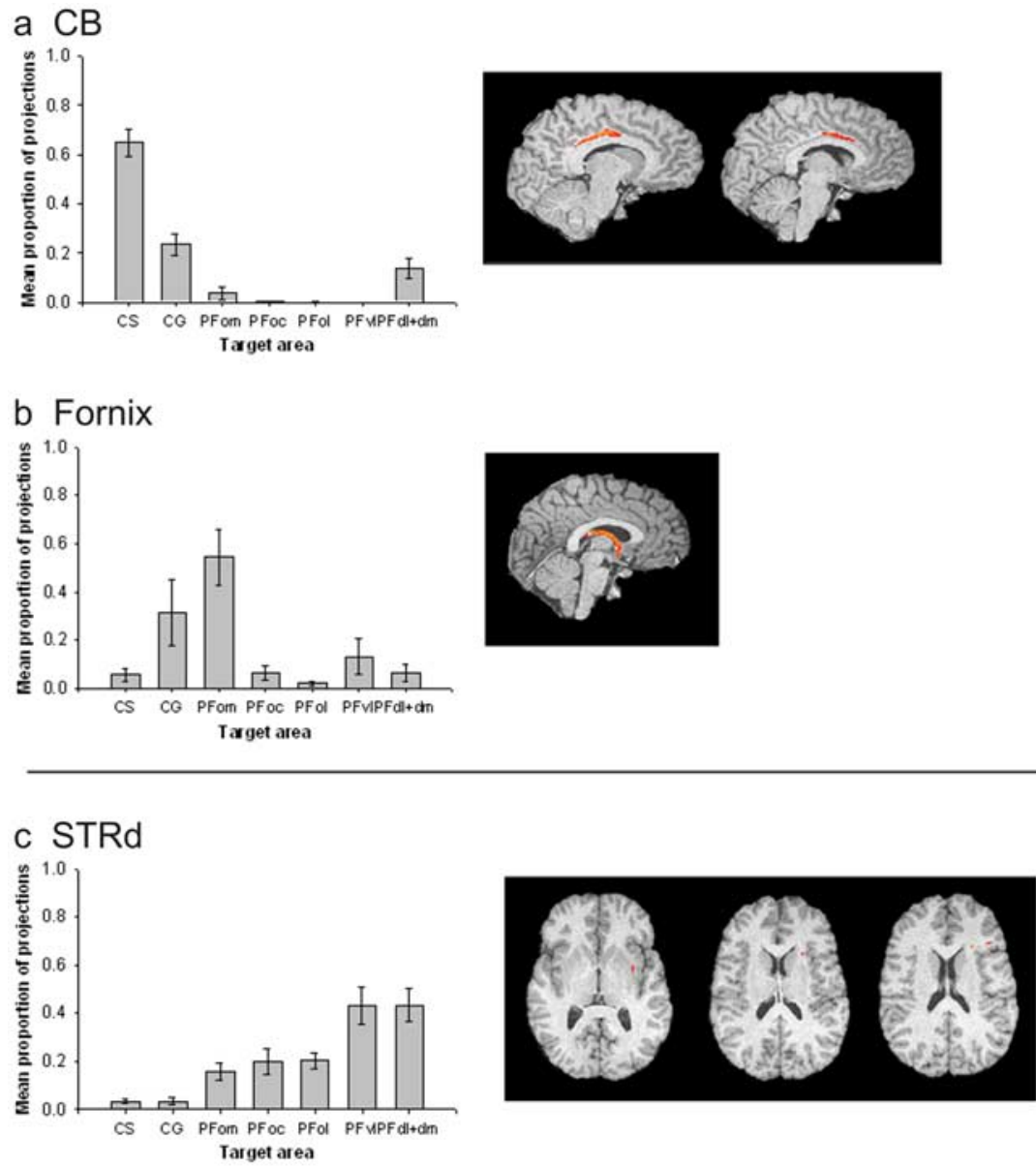

\section{d STRV}
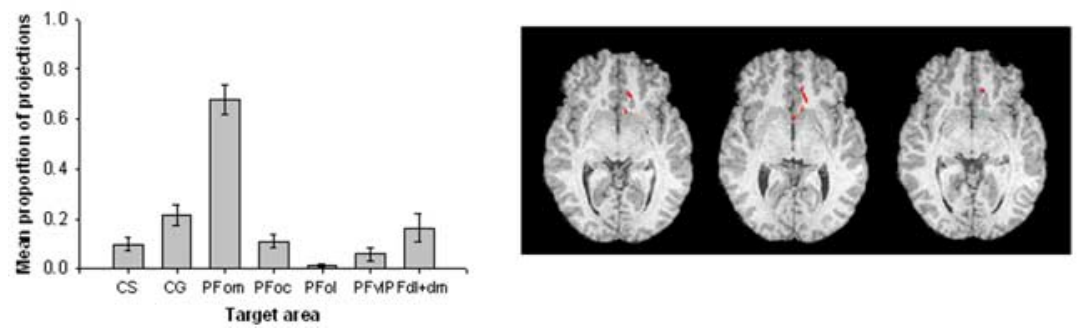

Figure 8. Quantitative results of probabilistic tractography from medial seed masks $(\boldsymbol{a})$ and striatal seed masks $(\boldsymbol{b})$ in the human brain. $\boldsymbol{a}$, The majority of connections of the CB are with CS and CG. The fornix has a high proportion of projections to PFom. Example tracts are shown for a single human subject. $\boldsymbol{b}$, STRd has relatively high connections in the PFd, PFdl $+d m$, and PFvl. The STRv has a stronger proportion of connections with PFom and also CG.

\section{Human PFC connections with medial} seed areas

The connections of two medial seed areas, the fornix and the $\mathrm{CB}$, with the eight PFC regions were compared (Fig. 8a). The significant interaction between seed area and target mask $\left(F_{(2.388,16.714)}=11.426 ; p<\right.$ $0.001)$ demonstrated that the two medial seed regions possessed distinct patterns of connections with the PFC.

Subsequent one-way ANOVAs demonstrated significant differences in the probability of connections between the fornix and the various $\mathrm{PFC}$ areas $\left(F_{(2.431}\right.$, 21.879) $=46.606 ; p<0.001)$. The human fornix had its greatest probability of connection with the PFom. A one-way ANOVA also demonstrated significant variation in the probability of PFC connections with the $\mathrm{CB}$. The highest connection probabilities for $\mathrm{CB}$ were with dorsomedial areas as opposed to ventromedial orbital areas, as was the case with the fornix. The highest probability of connection for $\mathrm{CB}$ was with the CS and to a lesser extent with CG $\left(F_{(1.744,12.205)}=5.548 ; p<\right.$ $0.05)$. Connections with the $\mathrm{PFdl}+\mathrm{dm}$ were also apparent.

\section{Human PFC connections with striatal} seed areas

Comparison of the PFC connections of the STRd and STRv seed areas (Fig. 8b) revealed a significant interaction between the connections of these two seed areas with the eight PFC areas in human subjects $\left(F_{(4.338,18.364)}=18.726 ; p<0.001\right)$. Analysis of the human STRd results indicated significant variation in the probability of its connections with the PFC; connections were most probable with the dorsal and lateral areas such as PFvl and $\mathrm{PFdl}+\mathrm{dm}$, and the highest probability connection was with the $\mathrm{PFdl}+\mathrm{dm}$ $\left(F_{(2.882,23.054)}=8.929 ; p<0.001\right)$.

The STR R had a much higher probability of connection with medial and orbital regions such as CS, CG, and most particularly PFom. Again, the variation in connection probabilities across the PFC areas was significant $\left(F_{(3.500,28.002)}=30.032\right.$; $p<0.001)$.

\section{Macaque PFC connections with lateral seed areas}

As was the case for the human PFC, there were significant differences in which macaque PFC areas showed the highest probability of connection to the lateral seed areas, with a significant interaction between the lateral seed area and PFC target area $\left(F_{(18.000}\right.$, $54.000)=6.736 ; p<0.001)$. The results are summarized in Figure 9. 


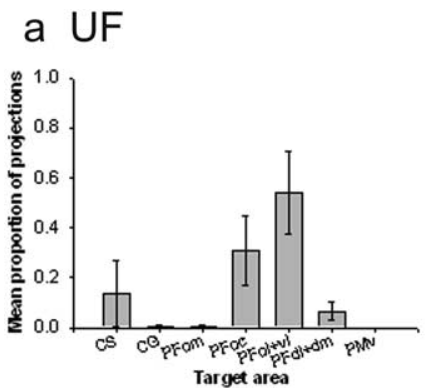

c SLF

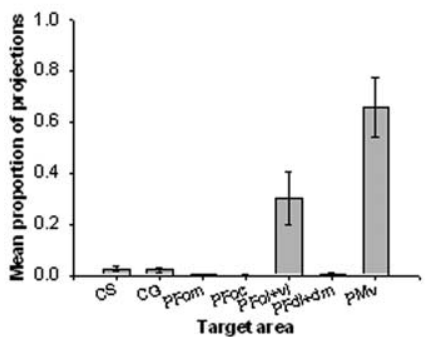

b EC

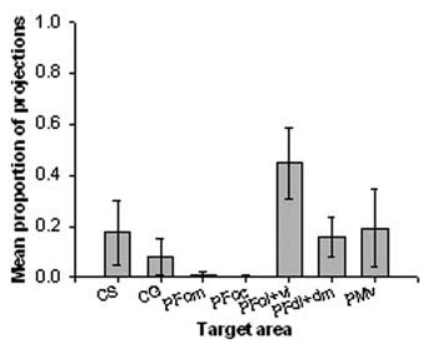

d Amygdala

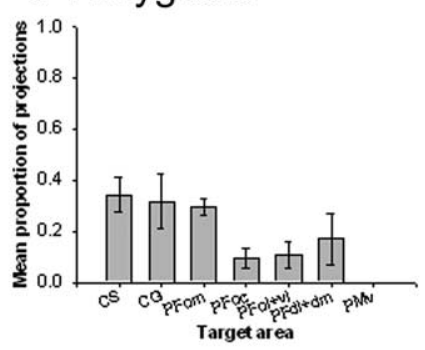

Figure 9. Quantitative results of probabilistic tractography from lateral seed masks in the macaque brain. $\boldsymbol{a}$, The majority of connections from anterior temporal lobe via the UF are to orbital PFC. $\boldsymbol{b}$, Connections from posterior temporal lobe areas via the $\mathrm{EC}$ are mainly to the lateral orbital and ventrolateral PFC areas. $c$, Connections via the SLF, connecting with parietal areas, are most likely to be with the PFvl and PMv. $\boldsymbol{d}$, The amygdala connections are most likely to be with medial PFC areas.

In the macaque, there was significant variation in the connection probability of the UF with the various PFC areas $\left(F_{(3.574}\right.$, $10.722)=3.807 ; p<0.005)$. The most probable UF connection was with PFoc and PFol+vl (Fig. 9a). The UF connection pattern with the PFC is similar in macaque and human species. The between-species comparison is hampered by the difficulty of defining corresponding areas in the macaque and human brain; for example, the macaque $\mathrm{PFol}+\mathrm{vl}$ area may resemble both the PFvl and PFol areas of the human brain. There was nevertheless a suggestion that, as in the human brain, the UF connections are biased toward more medial areas of the orbital cortex.

There was significant variation in the probability of the connections that the EC seed area made with the various $\mathrm{PFC}$ regions $\left(F_{(6.000,18.000)}=1.982\right.$; NS $)$. The foremost connection was with the PFvl, as was the case in the human brains (Fig. 9b). For both the UF and the EC, there was a suggestion that the human EC connections were more biased toward the medial and orbital surface than was the case in the macaque.

In the macaque brain, we were unable to reliably distinguish between the projections of the SLFII and SLFIII; the two parts of the SLF fascicle were therefore considered together. The SLF connections with the PFC regions varied significantly $\left(F_{(1.037,3.110)}=\right.$ 15.168; $p<0.05)$ and were most prominent with the PFol $+\mathrm{vl}$, and PMv (Fig. 9c). We noted however that there was also a large projection to the dorsal PFC region, but it was with a region just posterior to the $\mathrm{PFdl}+\mathrm{dm}$ mask that we had drawn. Thus, in both species, the SLF also projects to the PFdl $+\mathrm{dm}$ region, but many of the projections that were traceable with DWI tractography arrived near the posterior boundary of $\mathrm{PFdl}+\mathrm{dm}$ and close to the FEF region. If this boundary had been drawn slightly more posteriorly in the macaques, then there would have been a clearer quantitative correspondence between the SLF projections to the $\mathrm{PFdl}+\mathrm{dm}$ in the two species.

As was the case in the human brain, the probability of amygdala connections with various PFC areas varied significantly a

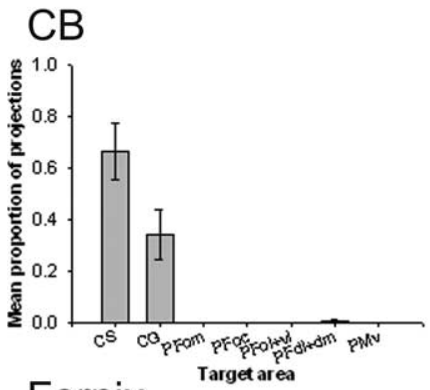

Fornix
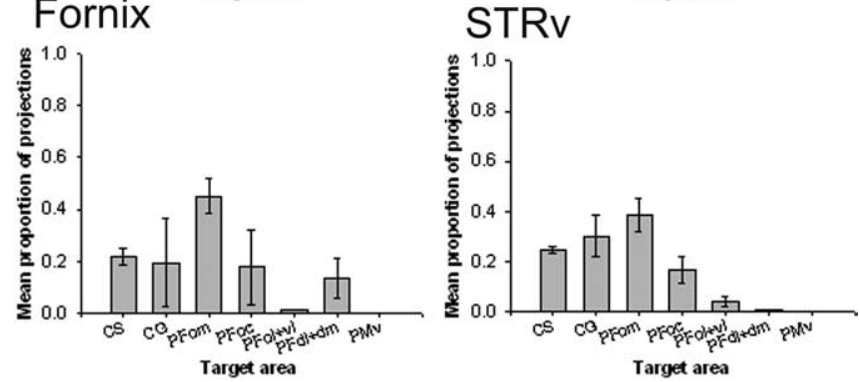

Figure 10. Quantitative results of probabilistic tractography from medial seed masks ( $\boldsymbol{a})$ and striatal seed masks $(\boldsymbol{b})$ in the macaque brain. $\boldsymbol{a}$, The majority of connections of the $\mathrm{CB}$ are with CS and CG, as in the human brains. The fornix has a high probability of connection with PFom, also similar to the human. $\boldsymbol{b}$, There is a relatively high probability connection between STRd and the PFdl $+d m$, as in the human, although this result is not so clear in the macaque brain, and high probability of cingulate projections is also indicated. The STRv has a higher probability of connections with PFom and also CG, and, again, this is clear in both species.

$\left(F_{(6.000,18.000)}=3.406 ; p<0.05\right)$. In the macaque, the main and most consistent connection was with the medial orbital PFom region (Fig. 9d), as was the case in the human subjects. In the macaque, however, connections with the more medial cingulate areas, CG and CS, also had high probability.

\section{Macaque PFC connections with medial seed areas}

The macaque PFC connections with the medial areas, fornix and $\mathrm{CB}$, were similar in pattern to that observed in the human brain (compare Figs. $8 a, 10 a$ ). Tracts seeded in the macaque fornix terminated mainly in the orbitofrontal cortex, CS and CG, with the highest probability of connection being with the PFom, as in the human. The majority of seeds from the macaque $\mathrm{CB}$ terminated in the CS and the CG to a lesser extent, again the same trend as in the human subjects. However, problems with the registration of one macaque brain to DWI space, and the added complication that the structural scan did not include all of the gray matter in the region of the fornix, made it necessary to discard the medial results for one animal, and the low sample size was not amenable to statistical analysis across the full set of eight PFC areas.

\section{Macaque PFC connections with striatal seed areas}

There were significant differences in the connections of the two striatal areas, STRv and STRd, with the different PFC regions $\left(F_{(6.000,18000)}=6.293 ; p<0.005\right)$. When STRd was considered in isolation, there was significant variation $\left(F_{(2.616,7.849)}=4.262 ; p<0.05\right)$ in the probabilities of its connections with each PFC region (Fig. $10 a)$. Like the human equivalent, there was a high probability of connection with $\mathrm{PFdl}+\mathrm{dm}$. There was also a high probability of connection to CG and CS, which was not seen in the human brains.

The connection probabilities of the macaque STRv to the PFC regions varied significantly $\left(F_{(2.601,7.804)}=9.572 ; p<0.05\right)$. The 
results are summarized in Figure 10b. As was the case in the human brains, the most probable connections were with the medial orbital region PFom, and the connections with the cingulate areas CG and CS were also strong.

\section{Discussion}

In the human, each subcortical region had a distinct pattern of connectivity with PFC areas, and each PFC area had a characteristic pattern of connectivity with subcortical areas and, by implication, with posterior cortical areas (Figs. 6-9). Although there may be a relative increase in white matter volume in human PFC (Schoenemann et al., 2005), patterns of human PFC connectivity resembled those found in macaque, in both the present DWI study and previous tracer studies. In the case of one human region, the POp, the connection pattern, however, was reminiscent of macaque PMv rather than PFC.

A meta-analysis of tracer investigations showed that, although macaque PFC areas share some connections, each has a unique connectivity profile or "fingerprint" (Passingham et al., 2002). The connectivity of each area determines its functional role in a network (Stephan et al., 2000; Kotter et al., 2001), and our results suggest that this is also true for human PFC.

Temporal-frontal connections are organized in a similar way in both species. The most probable connections of human UF were with areas PFoc and PFol, whereas the EC had a more lateral range centered on PFol and PFvl (Fig. 6). Although correspondences between PFC boundaries in the two species may not have been exact, a similar trend was discerned in the macaque; the most probable UF connections were with PFoc and PFol $+\mathrm{vl}$, and those of EC were with PFol+vl. Such patterns can be interpreted as follows. First, more anterior or inferior areas on the one hand and more posterior or superior temporal lobe areas on the other hand are, respectively, more likely to be interconnected with the PFC via the UF and EC (Goldman-Rakic et al., 1984; Petrides and Pandya, 1988, 2002b; Ungerleider et al., 1989). Second, moving anterior within the macaque temporal lobe, there is a decreasing tendency for PFvl connections and an increasing tendency for connections with more anterior and orbital PFC (GoldmanRakic et al., 1984; Petrides and Pandya, 1988; Webster et al., 1994; Carmichael and Price, 1995; Lavenex et al., 2002; Kondo et al., 2003) For example, posterior temporal-occipital area TEO connects with PFvl areas 8,45 , and 12 , but anterior temporal pole has strong connections with orbital PFC. Inferotemporal cortex area TE, with an intermediate temporal lobe position, has connections with PFvl centered on $12 \mathrm{l}$ but which extend into orbital PFC. The mid-portion of superior temporal auditory association areas are more likely to project to PFvl via the EC (Petrides and Pandya, 2002b).

Neuroimaging has implicated human PFvl in the selection of task-relevant information and memory retrieval for guiding decision and action selection (Buckner et al., 1995; Petrides et al., 1995). Lesion and single-cell data (Lebedev et al., 2004; Rushworth et al., 2004) suggest the same is true in the macaque, in which the UF has been shown to mediate these functions (Gutnikov et al., 1997).

The connections of the PFC with parietal cortex were assessed by analyzing two branches of the SLF. The human dorsomedial SLF (SLFII) was highly likely connected to PFdl $+\mathrm{dm}$, and these connections resemble the connections of parietal regions with dorsolateral prefrontal cortex in the macaque SLFII pathway (Petrides and Pandya, 2002b). The SLFII may, in both species, mediate similar interactions between posterior parietal cortex and PFdl $+\mathrm{dm}$ that occur with increasing working memory demands (Chafee and Goldman-Rakic, 2000; Sakai et al., 2002).

In the human brain, the other division of the SLF, SLFIII, was prominently linked with PFvl. In the macaque, SLFIII had some connections with PFvl+ol, but its strongest connections are with premotor area PMv posterior to the PFC proper as reported previously (Petrides and Pandya, 1984). It contains fibers that connect PMv with anterior parietal regions (Matelli et al., 1986; Cavada and Goldman-Rakic, 1989). The results suggest that a human region considered part of PFC has connections like macaque PMv or adjacent area 44.

To better identify which part of human PFvl had this unusual connectivity, we first compared the terminations of EC and SLF (Fig. 7a). Within PFvl, the SLFIII connection was more posterior, superior, and lateral than the EC connection. We then compared the POp subregion of PFvl (Amunts et al., 1999) with the remaining anterior PFvl. Most SLFIII connections were with POp, and most EC connections were with more anterior PFvl (Fig. 7b). In summary, the connections of human POp are reminiscent of macaque PMv and area 44. This is consistent with cytoarchitectonic and neuroimaging evidence for homology and functional similarities (Pandya and Yeterian, 1996; Iacoboni et al., 1999; Petrides and Pandya, 2002a; Rizzolatti et al., 2002; Binkofski and Buccino, 2004).

In both species, we found that the connections of the amygdala with the PFC had a greater medial bias toward the PFom than the connections of the EC and UF (Figs. $6 e, 9 d$ ). Strong interconnections have been reported between medial orbitofrontal cortex and amygdala in macaque (Porrino et al., 1981; Carmichael and Price, 1995). The macaque orbitofrontal cortex and amygdala interact in reward-guided behavior and emotion (Baxter et al., 2000; Bechara et al., 2003; Holland and Gallagher, 2004; Izquierdo and Murray, 2004). Our results suggest that similar connections mediate the interaction of human amygdala and orbitofrontal cortex during reward-guided learning (Gottfried et al., 2003). There was also a high probability of connection between STRv and the same orbitofrontal regions. Again, this was consistent with tracer studies (Haber et al., 1995). The human amygdala, orbitofrontal cortex, and STRv are sometimes coactive during reinforcement learning (O’Doherty, 2004).

Consistent with previous studies (Kunishio and Haber, 1994), we found strong connection probabilities between macaque STRv and cingulate areas. The same trend was present in the human data, although human connection probabilities with the cingulate were uniformly lower. STRv and anterior cingulate regions may mediate persistent responding and effort-based cost/ benefit decisions in humans and other mammals (Walton et al., 2002; Gusnard et al., 2003). Aside from anomalous connections with cingulate regions (high in macaque and low in humans), STRd connections were mainly with lateral PFC in both macaques and humans as described previously (Lehericy et al., 2004).

There was a high probability of fornix connections with PFom in both species. The distribution of human fornix connections had an even more medial bias than those of the amygdala. In macaques, the connections of the hippocampal subiculum, which are partly carried by the fornix (Cavada et al., 2000), have such a medial bias (Carmichael and Price, 1995). Transection of human or macaque fornix results in memory impairment (Gaffan and Gaffan, 1991; Charles et al., 2004), but the extent to which impairments are attributable to disruption of hippocampus and PFom interactions has not been tested by a crossed lesion. There 
is human neuroimaging evidence for the two regions coactivating during memory encoding (Frey and Petrides, 2002).

The CB was interconnected with more dorsomedial PFC regions than the fornix in both species. In the macaque, the $\mathrm{CB}$ provides a route between hippocampus and dorsomedial PFC (Goldman-Rakic et al., 1984; Morris et al., 1999). The functional contribution of the $\mathrm{CB}$ is not established in primates, but $\mathrm{CB}$ damage in the rodent leads to milder memory impairments (Aggleton et al., 1995). Our results suggest that similar CBmediated networks operate in both human and macaques.

There were two discrepancies between the results for the two species. First, there was a trend for connections to the orbital regions to be stronger in the human; this may have been attributable to positioning the head coil at some distance from orbitofrontal cortex in the macaque. More noticeably, there was a dearth of cingulate connections in the human and an overabundance in the macaque data. This may be attributable to a slight repositioning of the anterior cingulate cortex in humans, which may be more anterior and ventral relative to the genu of the corpus callosum than the macaque anterior cingulate cortex. Alternatively, it may be a consequence of the distorting influence of the high FA of the corpus callosum, which can decrease the probability of tracts connecting to the adjacent cingulate cortex in human subjects. Although absolute voxel sizes were smaller in the macaque, voxels were larger relative to brain size and thus more prone to partial volume effects (Parker et al., 2002). Voxels on the edges of cingulate gray matter might also contain callosal white matter leading to possible overestimation of macaque cingulate cortex connections. Such problems may be reduced by higher spatial resolution data and modeling of complex fiber architecture (Parker and Alexander, 2003; Tuch et al., 2003), but even still, diffusion tractography is unlikely to reveal connection directionality or the spatial detail obtained in tracer studies. Furthermore, the true relationship between diffusion characteristics and features of fiber pathways, such as packing density or myelination, remains incompletely understood (Beaulieu, 2002).

The results nevertheless demonstrate similar PFC circuits interacting via regionally specific connections with subcortical and cortical areas in both human and macaques and aid efforts to establish anatomical correspondences between species. The study also helps validate diffusion tractography approaches and confirms the utility of anatomical studies in nonhuman primates for understanding human brain function.

\section{References}

Aggleton JP, Neave N, Nagle S, Sahgal A (1995) A comparison of the effects of medial prefrontal, cingulate cortex, and cingulum bundle lesions on tests of spatial memory: evidence of a double dissociation between frontal and cingulum bundle contributions. J Neurosci 15:7270-7281.

Amaral DG, Insausti R (1990) Hippocampal function. In: The human nervous system, Chap 21 (Paxinos G, ed), pp 711-756. San Diego: Academic.

Amaral DG, Price JL (1984) Amygdalo-cortical projections in the monkey (Macaca fascicularis). J Comp Neurol 230:465-496.

Amunts K, Schleicher A, Burgel U, Mohlberg H, Uylings HB, Zilles K (1999) Broca's region revisited: cytoarchitecture and intersubject variability. J Comp Neurol 412:319-341.

Andersson J, Richter M, Richter W, Skare S, Nunes RG, Robson MD, Behrens TEJ (2004) Effects of susceptibility distortions on tractography. In: ISMRM Abstr. Kyoto, Japan.

Basser PJ, Jones DK (2002) Diffusion-tensor MRI: theory, experimental design and data analysis-a technical review. NMR Biomed 15:456-467.

Baxter MG, Parker A, Lindner CC, Izquierdo AD, Murray EA (2000) Control of response selection by reinforcer value requires interaction of amygdala and orbital prefrontal cortex. J Neurosci 20:4311-4319.

Beaulieu A (2002) A space for measuring mind and brain: interdisciplinary and digital tools in the development of brain mapping and functional imaging, 1980-1990. Brain Cogn 49:13-33.

Bechara A, Damasio H, Damasio AR (2003) Role of the amygdala in decision-making. Ann NY Acad Sci 985:356-369.

Behrens TE, Woolrich MW, Jenkinson M, Johansen-Berg H, Nunes RG, Clare S, Matthews PM, Brady JM, Smith SM (2003a) Characterization and propagation of uncertainty in diffusion-weighted MR imaging. Magn Reson Med 50:1077-1088.

Behrens TE, Johansen-Berg H, Woolrich MW, Smith SM, Wheeler-Kingshott CA, Boulby PA, Barker GJ, Sillery EL, Sheehan K, Ciccarelli O, Thompson AJ, Brady JM, Matthews PM (2003b) Non-invasive mapping of connections between human thalamus and cortex using diffusion imaging. Nat Neurosci 6:750-757.

Binkofski F, Buccino G (2004) Motor functions of the Broca's region. Brain Lang 89:362-369.

Buckner RL, Petersen SE, Ojemann JG, Miezin FM, Squire LR, Raichle ME (1995) Functional anatomical studies of explicit and implicit memory retrieval tasks. J Neurosci 15:12-29.

Carmichael ST, Price JL (1995) Limbic connections of the orbital and medial prefrontal cortex in macaque monkeys. J Comp Neurol 363:615-641.

Catani M, Howard RJ, Pajevic S, Jones DK (2002) Virtual in vivo interactive dissection of white matter fasciculi in the human brain. NeuroImage 17:77-94.

Cavada C, Goldman-Rakic PS (1989) Posterior parietal cortex in rhesus monkey. II. Evidence for segregated corticocortical networks linking sensory and limbic areas with the frontal lobe. J Comp Neurol 287:422-445.

Cavada C, Company T, Tejedor J, Cruz-Rizzolo RJ, Reinoso-Suarez F (2000) The anatomical connections of the macaque monkey orbitofrontal cortex. A review. Cereb Cortex 10:220-242.

Chafee MV, Goldman-Rakic PS (2000) Inactivation of parietal and prefrontal cortex reveals interdependence of neural activity during memoryguided saccades. J Neurophysiol 83:1550-1566.

Charles DP, Gaffan D, Buckley MJ (2004) Impaired recency judgments and intact novelty judgments after fornix transection in monkeys. J Neurosci 24:2037-2044.

Chiavaras MM, Petrides M (2000) Orbitofrontal sulci of the human and macaque monkey brain. J Comp Neurol 422:35-54.

Collins DL, Neelin P, Peters TM, Evans AC (1994) Automatic 3D intersubject registration of MR volumetric data in standardized Talairach space. J Comput Assist Tomogr 18:192-205.

Crosby EC, Humphrey T, Lauer EW (1962) White matter of the hemisphere, Pt 3. In: Correlative anatomy of the nervous system. New York: Macmillan.

Eblen F, Graybiel AM (1995) Highly restricted origin of prefrontal cortical inputs to striosomes in the macaque monkey. J Neurosci 15:5999-6013.

Ferry AT, Ongur D, An X, Price JL (2000) Prefrontal cortical projections to the striatum in macaque monkeys: evidence for an organization related to prefrontal networks. J Comp Neurol 425:447-470.

Frey S, Petrides M (2002) Orbitofrontal cortex and memory formation. Neuron 36:171-176.

Gaffan D, Gaffan EA (1991) Amnesia in man following transection of the fornix. A review. Brain 114:2611-2618.

Goldman-Rakic PS, Selemon LD, Schwartz ML (1984) Dual pathways connecting the dorsolateral prefrontal cortex with the hippocampal formation and parahippocampal cortex in the rhesus monkey. Neuroscience 12:719-743.

Gottfried JA, O’Doherty J, Dolan RJ (2003) Encoding predictive reward value in human amygdala and orbitofrontal cortex. Science 301:1104-1107.

Gusnard DA, Ollinger JM, Shulman GL, Cloninger CR, Price JL, Van Essen DC, Raichle ME (2003) Persistence and brain circuitry. Proc Natl Acad Sci USA 100:3479-3484.

Gutnikov SA, Ma YY, Gaffan D (1997) Temporo-frontal disconnection impairs visual-visual paired association learning but not configural learning in Macaca monkeys. Eur J Neurosci 9:1524-1529.

Haber SN, Kunishio K, Mizobuchi M, Lynd-Balta E (1995) The orbital and medial prefrontal circuit through the primate basal ganglia. J Neurosci 15:4851-4867.

Hagmann P, Thiran JP, Jonasson L, Vandergheynst P, Clarke S, Maeder P, Meuli R (2003) DTI mapping of human brain connectivity: statistical fibre tracking and virtual dissection. NeuroImage 19:545-554. 
Holland PC, Gallagher M (2004) Amygdala-frontal interactions and reward expectancy. Curr Opin Neurobiol 14:148-155.

Iacoboni M, Woods RP, Brass M, Bekkering H, Mazziotta JC, Rizzolatti G (1999) Cortical mechanisms of human imitation. Science 286:2526-2528.

Izquierdo A, Murray EA (2004) Combined unilateral lesions of the amygdala and orbital prefrontal cortex impair affective processing in rhesus monkeys. J Neurophysiol 91:2023-2039.

Jenkinson M, Smith S (2001) A global optimisation method for robust affine registration of brain images. Med Image Anal 5:143-156.

Johansen-Berg H, Behrens TE, Sillery E, Ciccarelli O, Thompson AJ, Smith SM, Matthews PM (2005) Functional-anatomical validation and individual variation of diffusion tractography-based segmentation of the human thalamus. Cereb Cortex 15:31-39.

Jones DK, Simmons A, Williams SC, Horsfield MA (1999) Non-invasive assessment of axonal fiber connectivity in the human brain via diffusion tensor MRI. Magn Reson Med 42:37-41.

Kondo H, Saleem KS, Price JL (2003) Differential connections of the temporal pole with the orbital and medial prefrontal networks in macaque monkeys. J Comp Neurol 465:499-523.

Kotter R, Stephan KE, Palomero-Gallagher N, Geyer S, Schleicher A, Zilles K (2001) Multimodal characterisation of cortical areas by multivariate analyses of receptor binding and connectivity data. Anat Embryol (Berl) 204:333-350.

Kunishio K, Haber SN (1994) Primate cingulostriatal projection: limbic striatal versus sensorimotor striatal input. J Comp Neurol 350:337-356.

Lavenex P, Suzuki WA, Amaral DG (2002) Perirhinal and parahippocampal cortices of the macaque monkey: projections to the neocortex. J Comp Neurol 447:394-420.

Lebedev MA, Messinger A, Kralik JD, Wise SP (2004) Representation of attended versus remembered locations in prefrontal cortex. PLoS Biol 2:e365.

Lehericy S, Ducros M, Van de Moortele PF, Francois C, Thivard L, Poupon C, Swindale N, Ugurbil K, Kim DS (2004) Diffusion tensor fiber tracking shows distinct corticostriatal circuits in humans. Ann Neurol 55:522-529.

Makris N, Kennedy DN, McInerney S, Sorensen AG, Wang R, Caviness Jr VS, Pandya DN (2005) Segmentation of Subcomponents within the superior longitudinal fascicle in humans: a quantitative, in vivo, DT-MRI study. Cereb Cortex 15:854-869.

Matelli M, Camarda R, Glickstein M, Rizzolatti G (1986) Afferent and efferent projections of the inferior area 6 in the macaque monkey. J Comp Neurol 251:281-298.

Matsumoto K, Suzuki W, Tanaka K (2003) Neuronal correlates of goalbased motor selection in the prefrontal cortex. Science 301:229-232.

Meredith GE, Pattiselanno A, Groenewegen HJ, Haber SN (1996) Shell and core in monkey and human nucleus accumbens identified with antibodies to calbindin-D28k. J Comp Neurol 365:628-639.

Mori S, Kaufmann WE, Davatzikos C, Stieltjes B, Amodei L, Fredericksen K, Pearlson GD, Melhem ER, Solaiyappan M, Raymond GV, Moser HW, van Zijl PC (2002) Imaging cortical association tracts in the human brain using diffusion-tensor-based axonal tracking. Magn Reson Med 47:215-223.

Morris R, Pandya DN, Petrides M (1999) Fiber system linking the middorsolateral frontal cortex with the retrosplenial/presubicular region in the rhesus monkey. J Comp Neurol 407:183-192.

Munger P, Crelier GR, Peters TM, Pike GB (2000) An inverse problem approach to the correction of distortion in EPI images. IEEE Trans Med Imaging 19:681-689.

O'Doherty JP (2004) Reward representations and reward-related learning in the human brain: insights from neuroimaging. Curr Opin Neurobiol 14:769-776.

Ongur D, Ferry AT, Price JL (2003) Architectonic subdivision of the human orbital and medial prefrontal cortex. J Comp Neurol 460:425-449.

Pandya DN, Yeterian EH (1996) Comparison of prefrontal architecture and connections. Philos Trans R Soc Lond B Biol Sci 351:1423-1432.

Parker GJ, Alexander DC (2003) Probabilistic Monte Carlo based mapping of cerebral connections utilising whole-brain crossing fibre information. Inf Process Med Imaging 18:684-695.

Parker GJ, Stephan KE, Barker GJ, Rowe JB, MacManus DG, WheelerKingshott CA, Ciccarelli O, Passingham RE, Spinks RL, Lemon RN, Turner R (2002) Initial demonstration of in vivo tracing of axonal projections in the macaque brain and comparison with the human brain using diffusion tensor imaging and fast marching tractography. NeuroImage 15:797-809.

Passingham RE (1995) The frontal lobes and voluntary action. New York: Oxford UP.

Passingham RE, Stephan KE, Kotter R (2002) The anatomical basis of functional localization in the cortex. Nat Rev Neurosci 3:606-616.

Paus T (1996) Location and function of the human frontal eye-field: a selective review. Neuropsychologia 34:475-483.

Paus T (2001) Primate anterior cingulate cortex: where motor control, drive and cognition interface. Nat Rev Neurosci 2:417-424.

Paus T, Otaky N, Caramanos Z, MacDonald D, Zijdenbos A, D’Avirro D, Gutmans D, Holmes C, Tomaiuolo F, Evans AC (1996) In vivo morphometry of the intrasulcal gray matter in the human cingulate, paracingulate, and superior-rostral sulci: hemispheric asymmetries, gender differences and probability maps. J Comp Neurol 376:664-673.

Paus T, Castro-Alamancos MA, Petrides M (2001) Cortico-cortical connectivity of the human mid-dorsolateral frontal cortex and its modulation by repetitive transcranial magnetic stimulation. Eur J Neurosci 14:1405-1411.

Petrides M (2005) Lateral prefrontal cortex: architectonic and functional organization. Philos Trans R Soc Lond B Biol Sci 360:781-795.

Petrides M, Pandya DN (1984) Projections to the frontal cortex from the posterior parietal region in the rhesus monkey. J Comp Neurol 228:105-116.

Petrides M, Pandya DN (1988) Association fiber pathways to the frontal cortex from the superior temporal region in the rhesus monkey. J Comp Neurol 273:52-66.

Petrides M, Pandya DN (2002a) Comparative cytoarchitectonic analysis of the human and the macaque ventrolateral prefrontal cortex and corticocortical connection patterns in the monkey. Eur J Neurosci 16:291-310.

Petrides M, Pandya DN (2002b) Association pathways of the prefrontal cortex and functional observations. In: Principles of frontal lobe function (Stuss DT, Knight RT, eds), pp 31-50. New York: Oxford UP.

Petrides M, Alivisatos B, Evans AC (1995) Functional activation of the human ventrolateral frontal cortex during mnemonic retrieval of verbal information. Proc Natl Acad Sci USA 92:5803-5807.

Picard N, Strick PL (2001) Imaging the premotor areas. Curr Opin Neurobiol 11:663-672.

Pinsk MA, Moore T, Richter MC, Gross CG, Kastner S (2005) Methods for functional magnetic resonance imaging in normal and lesioned behaving monkeys. J Neurosci Methods 143:179-195.

Porrino LJ, Crane AM, Goldman-Rakic PS (1981) Direct and indirect pathways from the amygdala to the frontal lobe in rhesus monkeys. J Comp Neurol 198:121-136.

Powell HW, Guye M, Parker GJ, Symms MR, Boulby P, Koepp MJ, Barker GJ, Duncan JS (2004) Noninvasive in vivo demonstration of the connections of the human parahippocampal gyrus. NeuroImage 22:740-747.

Procyk E, Joseph JP (2001) Characterization of serial order encoding in the monkey anterior cingulate sulcus. Eur J Neurosci 14:1041-1046.

Rajkowska G, Goldman-Rakic PS (1995) Cytoarchitectonic definition of prefrontal areas in the normal human cortex. II. Variability in locations of areas 9 and 46 and relationship to the Talairach coordinate system. Cereb Cortex 5:323-337.

Rizzolatti G, Fogassi L, Gallese V (2002) Motor and cognitive functions of the ventral premotor cortex. Curr Opin Neurobiol 12:149-154.

Rushworth MF, Walton ME, Kennerley SW, Bannerman DM (2004) Action sets and decisions in the medial frontal cortex. Trends Cogn Sci 8:410-417.

Sakai K, Rowe JB, Passingham RE (2002) Active maintenance in prefrontal area 46 creates distractor-resistant memory. Nat Neurosci 5:479-484.

Schoenemann PT, Sheehan MJ, Glotzer LD (2005) Prefrontal white matter volume is disproportionately larger in humans than in other primates. Nat Neurosci 8:242-252.

Selemon LD, Goldman-Rakic PS (1985) Longitudinal topography and interdigitation of corticostriatal projections in the rhesus monkey. J Neurosci 5:776-794.

Selemon LD, Goldman-Rakic PS (1988) Common cortical and subcortical targets of the dorsolateral prefrontal and posterior parietal cortices in the rhesus monkey: evidence for a distributed neural network subserving spatially guided behavior. J Neurosci 8:4049-4068.

Smith CG (1971) The cerebral hemisphere III: internal structure. In: Basic neuroanatomy, Ed 2. Toronto: University of Toronto. 
Smith SM (2002) Fast robust automated brain extraction. Hum Brain Mapp 17:143-155

Stephan KE, Hilgetag CC, Burns GA, O’Neill MA, Young MP, Kotter R (2000) Computational analysis of functional connectivity between areas of primate cerebral cortex. Philos Trans R Soc Lond B Biol Sci 355:111-126.

Stieltjes B, Kaufmann WE, van Zijl PC, Fredericksen K, Pearlson GD, Solaiyappan M, Mori S (2001) Diffusion tensor imaging and axonal tracking in the human brainstem. NeuroImage 14:723-735.

Talairach J, Tournoux P (1988) Co-planar stereotaxic atlas of the human brain. Stuttgart, Germany: Thieme.

Tournier JD, Calamante F, Gadian DG, Connelly A (2003) Diffusionweighted magnetic resonance imaging fibre tracking using a front evolution algorithm. NeuroImage 20:276-288.

Tuch DS, Reese TG, Wiegell MR, Wedeen VJ (2003) Diffusion MRI of complex neural architecture. Neuron 40:885-895.

Ungerleider LG, Gaffan D, Pelak VS (1989) Projections from inferior temporal cortex to prefrontal cortex via the uncinate fascicle in rhesus monkeys. Exp Brain Res 76:473-484.

Van Hoesen GW, Morecraft RJ, Vogt BA (1993) Connections of the monkey cingulate cortex. In: Neurobiology of cingulate cortex and limbic thalamus (Vogt BA, Gabriel M, eds). Boston: Birkhauser.

Vogt BA, Nimchinsky EA, Vogt LJ, Hof PR (1995) Human cingulate cortex: surface features, flat maps, and cytoarchitecture. J Comp Neurol 359:490-506.

Vogt BA, Berger GR, Derbyshire SW (2003) Structural and functional dichotomy of human midcingulate cortex. Eur J Neurosci 18:3134-3144.

Von Bonin G, Bailey P, eds (1947) The neocortex of Macaca mulatta. Urbana, IL: University of Illinois.

Voorn P, Vanderschuren LJ, Groenewegen HJ, Robbins TW, Pennartz CM (2004) Putting a spin on the dorsal-ventral divide of the striatum. Trends Neurosci 27:468-474.

Walton ME, Bannerman DM, Rushworth MF (2002) The role of rat medial frontal cortex in effort-based decision making. J Neurosci 22:10996-11003.

Walton ME, Devlin JT, Rushworth MF (2004) Interactions between decision making and performance monitoring within prefrontal cortex. Nat Neurosci 7:1259-1265

Webster MJ, Bachevalier J, Ungerleider LG (1994) Connections of inferior temporal areas TEO and TE with parietal and frontal cortex in macaque monkeys. Cereb Cortex 4:470-483.

Weniger G, Boucsein K, Irle E (2004) Impaired associative memory in temporal lobe epilepsy subjects after lesions of hippocampus, parahippocampal gyrus, and amygdala. Hippocampus 14:785-796.

Zhang Y, Brady M, Smith S (2001) Segmentation of brain MR images through a hidden Markov random field model and the expectationmaximization algorithm. IEEE Trans Med Imaging 20:45-57. 